\title{
LA COMUNICACIÓN INTEGRADA DE MARKETING (CIM) EN LA EDUCACION SUPERIOR (ES) EN TIEMPOS DE PANDEMIA
}

\section{INTEGRATED MARKETING COMMUNICATION (IMC) IN HIGHER EDUCATION (HE) IN PANDEMIC TIMES}

Janire Gordon-Isasi (Universidad de Deusto) ${ }^{1}$

Lorea Narvaiza Cantin (Universidad de Deusto) ${ }^{2}$

Juan José Gibaja Martíns (Universidad de Deusto) ${ }^{3}$

\section{Resumen:}

El artículo presenta la evolución de la comunicación integrada de marketing (CIM) desde sus inicios hacia un concepto que, tras adaptarse al ecosistema de marketing y comunicación actual, incluye estrategias digitales. Se pretende aplicar y adaptar la CIM al sector servicios y, más en concreto, al sector de la educación superior (ES), que ha sido escasamente estudiado hasta la fecha. El actual contexto sugiere también tener en cuenta la pandemia debida al COVID-19 y las posibles implicaciones que esta ha generado en el marketing de la educación superior (MES). La metodología seguida ha sido la de la revisión sistemática de la literatura sobre CIM en la ES. Las conclusiones de esta investigación indican que se requiere seguir investigando en materia de CIM aplicada al sector de la ES, ya que los beneficios generados pueden ser muy interesantes para las estrategias llevadas a cabo en el MES. A su vez, la originalidad del presente manuscrito reside en que se pretende mostrar el escenario actual del MES en la ES, en tiempos de pandemia y bajo el paraguas del enfoque CIM. Se centra en la interactividad como dimensión clave en las estrategias CIM y en el sector de la ES. Asimismo, se enfatiza la relevancia de la interacción con objeto de fidelizar a la audiencia en escenarios globales como la COVID-19.

Palabras clave: Comunicación integrada de marketing (CIM), marketing en la educación superior (MES), educación superior (ES), pandemia, sector servicios.

Códigos JEL: M31 Marketing.

\section{Resumen:}

The article presents the evolution of integrated marketing communication (IMC) from its beginnings towards a concept that, after adapting to the current marketing and communication ecosystem, includes digital strategies. It is intended to apply and adapt the IMC to the service sector and more specifically, to the higher education (HE) sector that has been scarcely studied to date. The current context also suggests taking into account the pandemic due to COVID-19 and the possible implications it has generated in the higher education marketing (HEM). The methodology followed has been a systematic literature review on IMC in HE. The conclusions of this research indicate that further research on IMC applied to the HE sector is required, since the benefits generated can be very interesting for the strategies carried out in the HEM. In turn, the originality of this manuscript resides in the fact that it is intended to show the current HEM

\footnotetext{
1 janiregordon@deusto.es, Universidad de Deusto.

2 lorea.narvaiza@deusto.es, Universidad de Deusto.

3 jjgibaja@deusto.es, Universidad de Deusto.

Recibido: 30 de octubre de 2020. Aceptado: 30 de noviembre de 2020.
} 
scenario in the HE, in times of pandemic and under the umbrella of the IMC approach. It focuses on interactivity as a key dimension in IMC strategies and in the HE sector. Likewise, the relevance of interaction is emphasized in order to foster durable relationships with the audience under the impact of global issues such as COVID-19.

Keywords: integrated marketing communication (IMC), higher education marketing (HEM), higher education (HE), pandemic, service sector.

JEL Codes: M31 Marketing.

\section{INTRODUCCIÓN}

La comunicación integrada de marketing (CIM) se entiende como la coordinación total de las comunicaciones y medios de marketing utilizados en las organizaciones (Kliatchko y Schultz, 2014; Luxton et al., 2017; Porcu et al., y Crespo-Almendros, 2019). En concreto según Porcu et al. (2017) se trata de un proceso interactivo centrado en los grupos de interés de la organización que fomenta las relaciones a corto plazo y el diálogo continuo.

En la actualidad las organizaciones utilizan varios canales y puntos de contacto. Se considera un punto de contacto cualquier situación en la que la organización y la audiencia interactúan. En las estrategias de la CIM, los puntos de contacto pueden estar controlados por las organizaciones o por los grupos de interés (Vollero et al., 2019), es decir, ambas partes son capaces de crear la interacción. Estos canales y puntos de contacto requieren ser gestionados con precisión por las organizaciones y son vitales en los servicios (Duncan y Moriarty, 2006). A pesar de su importancia, es escasa la literatura sobre la planificación y ejecución de estrategias de la CIM cuando intervienen diferentes medios de comunicación. En este sentido, son pocas las propuestas académicas que definen una guía o estructura que facilite el desarrollo de las estrategias CIM en las organizaciones. La falta de propuestas en la materia anterior puede ser debido a que hasta hace poco, las estrategias de la CIM no solían centrarse en actividades de comunicación bidireccional donde las organizaciones y también los consumidores crean contenidos en los medios (Aspara et al., 2014). Además, hasta donde sabemos, la CIM no se ha estudiado en profundidad en contextos digitales (Vollero et al., 2019). Sin embargo, considerando que en la era actual la audiencia utiliza múltiples medios digitales interactivos, es importante considerar dichos canales, medios y puntos de contacto con la audiencia cuando se implementa la estrategia de comunicación y marketing. De hecho, aunque la CIM es un concepto ampliamente aceptado en el entorno académico y profesional, la literatura muestra que ha estado rodeado de inconsistencia, lo que ha supuesto dificultades a la hora de definir el concepto, su practicidad y, por lo tanto, su implementación efectiva.

A lo largo de los años se han propuesto varias definiciones sobre la CIM (Kerr et al., 2008; Kliatchko, 2005; Porcu et al., 2017). También han sido objeto de investigación las dimensiones, los sistemas de medición (escalas), el enfoque desde el que se estudia el constructo (desde un enfoque 'amplio' que engloba todos los departamentos de una organización o desde un enfoque más reducido en la organización) (Porcu et al., 2019), su implementación (Manoli y Hodgkinson, 2019) y, además, su rendimiento (Luxton et al., 2017; Taylor, 2010). En consecuencia, algunos autores concluyen que se requieren más estudios empíricos sobre la CIM, principalmente centrados en la medición (Kitchen, 2017a), en el rendimiento (Porcu et al., 2019), en la implementación y en el componente digital (Vollero et al., 2019). Todo ello dará lugar a una efectiva interacción y comunicación bidireccional entre la organización y la audiencia.

Considerando lo mencionado en anteriores párrafos, el presente artículo pretende cubrir tres objetivos. En primer lugar, se presenta una revisión sistemática de la literatura en la que se 
estudia la evolución del enfoque de la CIM desde sus inicios hasta la incorporación de un enfoque más digital donde la interactividad ha cobrado mayor relevancia. En segundo lugar, se analiza el sector de la educación superior (ES) desde la perspectiva de la CIM y el marketing en la educación superior (MES). Finalmente, se expone el estado actual de la CIM en un contexto poco investigado, la educación superior, y bajo los efectos de la pandemia causada por la COVID-19 en el año 2020. La metodología seguida ha sido la de realizar la revisión de la literatura sobre CIM en la ES. Para ello se han realizado búsquedas sobre los términos antes citados en revistas indexadas en las bases de datos Web of Science (WOS) y Scopus.

\section{LA COMUNICACIÓN INTEGRADA DE MARKETING (CIM) Y SU EVOLUCIÓN}

La CIM es un concepto que posee una creciente relevancia para la comunidad académica y profesional. Muestra de ello es que desde 2016, una de las prioridades ha sido la necesidad de contar con enfoques integrados en las acciones de marketing (Marketing Science Institute, 2016). Más tarde, el Marketing Science Institute (2018) propuso dos prioridades de investigación. Primero, se recomendaba explorar sobre cómo las campañas y los mensajes deben integrarse entre diferentes plataformas y dispositivos. En segundo lugar, también se destacó la necesidad de seguir investigando en el diseño de campañas de comunicación omnicanal y su análisis de resultados. De hecho, la propuesta hacia una gestión eficiente de múltiples canales de manera coherente y homogénea (enfoque omnicanal) sigue siendo una prioridad de investigación para 2020 según el Marketing Science Institute (2020).

Otra evidencia que muestra la relevancia de la CIM es que se han dedicado varios números especiales a la misma a lo largo de los años (Kitchen, 2017b; Schultz y Schultz, 1998; Schultz y Patti, 2009; William A. Cook, 2004).

La definición de CIM se propuso en el año 1989 por la Asociación Americana de Agencias de Publicidad (4As) (Duncan y Caywood, 1996). Fue determinada como un concepto de planificación de marketing que integraba y combinaba varias disciplinas con el fin de proporcionar el máximo impacto en la comunicación por medio de la claridad y la coherencia. Más adelante, se fueron introduciendo nociones acerca del valor añadido que la CIM proporcionaba (Caywood et al., 1991). Uno de los elementos que los investigadores incluyeron en las primeras definiciones sobre la CIM se relacionaba con las reacciones de la audiencia (clientes actuales, potenciales y grupos de interés) ya que son relevantes para desarrollar estrategias de CIM efectivas. La interacción con la audiencia permite ayudar a mantener las relaciones con la misma y convertirla en prescriptora y/o 'leal' a la organización (Duncan, 1994; Nowak y Phelps, 1994; Schultz y Schultz, 1998; Schultz, 1991). En sugerencias posteriores, se mencionó la medición del constructo CIM (Schultz y Schultz, 2003). A lo largo de los años, la inclusión de una amplia variedad de disciplinas de la comunicación también ganó importancia en las estrategias del concepto (Percy, 2008; Kliatchko y Schultz, 2014; Séric et al., 2015; Porcu et al., 2017).

Considerando que la CIM es una estrategia aplicada en las organizaciones y una de sus grandes misiones es realizar comunicaciones de 'marca', se recomienda aplicar varios medios y canales de comunicación. Al mismo tiempo, se deben considerar los grupos de interés externos e internos (Kliatchko, 2008; Schultz y Schultz, 2003; Séric et al., 2015). Además, la World Wide Web fue lanzada en 1989 y desde entonces se ha fomentado la comunicación bidireccional, se han llevado a cabo estrategias centradas en la audiencia (Kliatchko, 2005), también se han aplicado múltiples canales en las estrategias (Kliatchko, 2005; Percy, 2008) y se ha publicado contenido significativo para fomentar la interacción (Kliatchko, 2008). 
A continuación, se muestra un resumen de las ultimas definiciones del constructo CIM propuestas del año 2005 al 2017.

\section{TABLA 1. ULTIMAS DEFINICIONES PROPUESTAS SOBRE IMC DEL AÑO 2005} AL 2017

\begin{tabular}{|c|c|c|}
\hline Autor & Fecha & Definición de la comunicación integrada de marketing (CIM) \\
\hline Kliatchko & 2005 & $\begin{array}{l}\text { CIM es el concepto y proceso de administrar estratégicamente programas de comunicación de marca } \\
\text { centrados en la audiencia, centrados en el canal y orientados a los resultados a lo largo del tiempo. } \\
\text { CIM, en su forma práctica, intenta combinar, integrar y sinergizar diferentes elementos de la combinación de } \\
\text { comunicaciones, ya que las fortalezas de una se utilizan para compensar las debilidades de otras. }\end{array}$ \\
\hline Kliatchko & 2008 & $\begin{array}{l}\text { CIM es un proceso impulsado por la audiencia para gestionar estratégicamente las partes interesadas, el } \\
\text { contenido, los canales y los resultados de los programas de comunicación de la marca. }\end{array}$ \\
\hline $\begin{array}{l}\text { Luck y } \\
\text { Moffatt }\end{array}$ & 2009 & $\begin{array}{l}\text { Es más que un proceso o actividad dentro de una organización: es un sistema de creencias y compromiso, } \\
\text { incrustado en la cultura de una organización, respaldado por la comunicación e impulsado por la tecnología y } \\
\text { la alta dirección. }\end{array}$ \\
\hline $\begin{array}{l}\text { Šerić y Gil- } \\
\quad \text { Saura }\end{array}$ & 2012 & $\begin{array}{c}\text { IMC es un proceso táctico y estratégico que fomenta sinergias entre diferentes canales de comunicación. Los } \\
\text { mensajes que se transmiten son claros y coherentes y la comunicación se centra en las tecnologías de la } \\
\text { información y la comunicación (TIC), base de datos y clientes. El IMC es clave para el valor y las relaciones } \\
\text { de marca. }\end{array}$ \\
\hline $\begin{array}{l}\text { Porcu, del } \\
\text { Barrio-García } \\
\text { y Kitchen }\end{array}$ & 2012 & $\begin{array}{c}\text { El proceso interactivo y sistémico de planificación transversal y optimización de mensajes a los grupos de } \\
\text { interés con el objetivo de comunicar con coherencia y transparencia para lograr sinergias y fomentar } \\
\text { relaciones rentables a corto, medio y largo plazo. }\end{array}$ \\
\hline $\begin{array}{l}\text { Kliatchko y } \\
\text { Schultz }\end{array}$ & 2014 & $\begin{array}{l}\text { En el estudio realizado por estos autores este es el resultado de su intento de explicar el concepto de IMC en } \\
\text { cuatro nociones para los miembros entrevistados por el cliente y la agencia (desde su punto de vista): } \\
\text { Uso de múltiples canales, puntos de contacto o puntos de contacto para entregar un mensaje a una audiencia } \\
\text { objetivo. } \\
\text { Centrado en el consumidor: es decir, la necesidad de una comprensión profunda del consumidor, } \\
\text { compromisos con el consumidor y conexiones y conversaciones continuas como base para la planificación y } \\
\text { ejecución de las comunicaciones de marketing. } \\
\text { Coordinación y consistencia de soluciones de comunicaciones } 360 \text { o totales. } \\
\text { Implicación del proceso empresarial general, no solo de las comunicaciones de marketing. }\end{array}$ \\
\hline $\begin{array}{l}\text { Šerić, Gil- } \\
\text { Saura y } \\
\text { Ozretić- } \\
\text { Došen }\end{array}$ & 2015 & $\begin{array}{l}\text { La comunicación de marketing integrada (IMC) es un proceso empresarial táctico y estratégico centrado en } \\
\text { el consumidor impulsado por los avances en las tecnologías de la información y la comunicación (TIC). } \\
\text { Entrega un mensaje claro y consistente a través de la coordinación y sinergias de diferentes herramientas y } \\
\text { canales de comunicación, con el fin de nutrir relaciones rentables y duraderas con los clientes y otras partes } \\
\text { interesadas y crear y mantener el valor de la marca. }\end{array}$ \\
\hline $\begin{array}{l}\text { Luxton, Reid } \\
\text { y Mavondo }\end{array}$ & 2017 & $\begin{array}{l}\text { Un mecanismo de implementación relacionado con el mercado de toda la empresa que permite la } \\
\text { optimización de los enfoques de comunicación para lograr una eficacia de comunicación superior. }\end{array}$ \\
\hline $\begin{array}{l}\text { Porcu, del } \\
\text { Barrio-García } \\
\text { y Kitchen }\end{array}$ & 2017 & $\begin{array}{l}\text { CIM es el proceso interactivo centrado en las partes interesadas de planificación interfuncional y alineación } \\
\text { de procesos organizativos, analíticos y de comunicación, que permite la posibilidad de un diálogo continuo } \\
\text { mediante la transmisión de mensajes coherentes y transparentes a través de todos los medios para fomentar } \\
\text { relaciones rentables a largo plazo que creen valor. }\end{array}$ \\
\hline
\end{tabular}

Desde que se propuso por primera vez a finales de la década de los ochenta, se consideró un enfoque holístico de marketing y comunicación, cuya principal capacidad era proporcionar respuestas efectivas a través de los diferentes medios que utilizan las organizaciones. La comunicación integrada de marketing (CIM) abarca todos los medios de marketing y 
comunicación en las organizaciones y de manera consistente (Daszkiewicz y Pukas, 2016; Porcu et al., 2017). No obstante, desde que la CIM se definió por primera vez a finales de la década de los ochenta por la Asociación Estadounidense de Agencias de Publicidad (4A's), se puede observar como se han sugerido varias propuestas en relación al concepto (Eagle y Brennan, 2007).

A su vez, a lo largo de los años, muchos autores han extraido ciertas dimensiones de las definiciones propuestas. Estas dimensiones concluyen que la CIM es un constructo multidimensional. La siguiente tabla (Tabla 2) muestra las dimensiones del constructo CIM propuestas del año 2005 al 2017.

\section{TABLA 2. ULTIMAS DIMENSIONES PROPUESTAS SOBRE CIM DEL AÑO 2005} AL 2017

\begin{tabular}{|c|c|c|}
\hline Autor & Fecha & Dimensiones de la comunicación integrada de marketing (CIM) \\
\hline Kliatchko & 2005 & $\begin{array}{l}\text { 1. Enfocado en la audiencia: múltiples mercados } \\
\text { 2.Centrado en canales: múltiples canales } \\
\text { 3. Orientado a resultados: medición financiera }\end{array}$ \\
\hline Reid & 2005 & $\begin{array}{l}\text { 1. Interactividad } \\
\text { 2. Marketing de misión } \\
\text { 3. Planificación y evaluación. }\end{array}$ \\
\hline Kliatchko & 2008 & $\begin{array}{c}\text { 1. Partes interesadas (orientación) } \\
\text { 2. Contenido } \\
\text { 3. Canal } \\
\text { 4. Resultados (medibles) } \\
\end{array}$ \\
\hline Moriarty y Schultz & 2012 & $\begin{array}{l}\text { 1. Comunicación interactiva } \\
\text { 2. Consistencia del mensaje } \\
\text { 3. Enfoque de marca } \\
\text { 4. Sinergia } \\
\text { 5. Orientación al cliente } \\
\text { 6. Integración perceptual } \\
\text { 7. Partes interesadas } \\
\text { 8. Relaciones } \\
\text { 9. Reciprocidad } \\
\text { 10. Puntos de contacto } \\
\text { 11. Gestión multifunctional } \\
\text { 12. Planificación y supervisión continuas }\end{array}$ \\
\hline $\begin{array}{l}\text { Porcu, Del Barrio- } \\
\text { García y Kitchen }\end{array}$ & 2012 & $\begin{array}{l}\text { 1. Única voz } \\
\text { 2. Interactividad } \\
\text { 3. Planificación multifuncional } \\
\text { 4. Relaciones rentables a largo plazo }\end{array}$ \\
\hline Syahputra & 2012 & $\begin{array}{l}\text { 1. Interactividad } \\
\text { 2. Comercialización de la misión } \\
\text { 3. Infraestructura organizativa } \\
\text { 4. Coherencia estratégica } \\
\text { 5. Planificación y evaluación }\end{array}$ \\
\hline Šerić y Gil-Saura & 2012 & $\begin{array}{l}\text { 1. Sinergias entre herramientas de comunicación } \\
\text { 2. Comunicación centrada en las TIC } \\
\text { 3. Consistencia del mensaje } \\
\text { 4. Comunicación centrada en el consumidor } \\
\text { 5. CIM como componente de la propuesta de valor de la marca }\end{array}$ \\
\hline $\begin{array}{l}\text { Porcu, Del Barrio- } \\
\text { García y Kitchen }\end{array}$ & 2017 & $\begin{array}{l}\text { 1. Consistencia del mensaje } \\
\text { 2. Interactividad } \\
\text { 3. Enfoque estratégico centrado en las partes interesadas } \\
\text { 4. Alineación organizativa }\end{array}$ \\
\hline
\end{tabular}

Fuente: elaboración propia a partir de varios autores.

Se puede observar como las dimensiones han evolucionado en consecuencia con las definiciones propuestas y también considerando los cambios vividos en el paradigma de marketing y comunicación. Se aprecia cómo tras las definiciones propuestas por Kliatchko en 2005 y 2008 se sugieren ciertas dimensiones, tres y cuarto respectivamente (ver tabla 2). 
Inicialmente en 2005, Kliatchko expuso tres dimensiones, la primera relacionada con el enfoque hacía la audiencia, la segunda centrada en canales y, por último, la dimensión orientada a resultados. Debido a la evolución del constructo CIM, en 2008 Klitachko adaptó la definición propuesta en 2005 (ver tabla 1) y extrajo cuatro dimensiones. La primera relacionada con la orientación hacía las partes interesadas de las organizaciones, la segunda centrada en el contenido, la tercera en el canal y la cuarta en la medición de los resultados del constructo CIM.

Otros autores como Šerić y Gil-Saura (2012) y Porcu, del Barrio-García y Kitchen (2017), también propusieron dimensiones para el constructo CIM tras formular una definición para el mismo. En el caso de estas últimas propuestas, se observa como se incluyen como dimensiones aspectos como la comunicación centrada en las tecnologías de la información y la comunicación (TIC) o la interactividad, respectivamente. Lo anterior puede ser debido al auge de los medios digitales. En cuanto a la interactividad, se observa como varios autores han incluido esta dimensión a lo largo de los años (ej. Reid, 2005; Porcu et al., 2012; Syahputra, 2012; Porcu et al., 2017). Los medios digitales son una gran ventaja a la hora de acometer dicha dimensión, ya que la interacción es llevada a cabo de manera más ágil gracias a las funcionalidades proporcionadas por dichos medios digitales. Esta tipología de medios es además aplicable a muchos sectores de actividad, especialmente al sector servicios.

Y es que la interactividad ha sido uno de los aspectos que más ha cambiado en los últimos tiempos en el marco de la CIM. Esta se ha considerado por algunos autores (Duncan y Moriarty, 1998) como un elemento clave de la integración en la comunicación. La interactividad permite una comunicación bidireccional simétrica como condición necesaria para un diálogo entre la marca y sus grupos de interés, clave según la definición y dimensiones propuestas por Porcu et al. (2017). En este sentido, las tecnologías digitales juegan en la actualidad un papel clave en la implementación de un enfoque integrado de la comunicación (Kliatchko y Schultz, 2014) ya que permiten maximizar cada elemento de la interactividad al combinar, por ejemplo, las redes sociales con los teléfonos móviles, y así permiten un nivel de ubicuidad en la comunicación sin precedentes. Finalmente, la pandemia de la COVID-19 ha acelerado los procesos de digitalización de las empresas para poder acceder a los clientes, y por ello, también ha puesto de relieve la importancia de CIM en la nueva normalidad. Todo lo anterior confirma que el interés sobre la CIM sigue creciendo tanto en el entorno académico como en el profesional.

Tal y como muestran las definiciones anteriores y también la incorporación de diferentes elementos a lo largo de los años, el abanico de medios de comunicación digitales disponibles se ha ampliado de manera significativa en las estrategias CIM. Todo ello, ha posibilitado la interacción con la audiencia, pero dificulta gestionar de manera efectiva las estrategias de la CIM en las organizaciones, dado que hay que realizar un seguimiento de todos los medios e interacciones en las que se está presente. Además, actualmente la comunicación interactiva es crucial entre las organizaciones y sus diferentes grupos de interés para sobrevivir, por ello, esta comunicación bidireccional se debe llevar a cabo de la mejor manera posible.

\subsection{CIM, la era digital y la interactividad}

Considerando el amplio espectro de medios disponibles, se puede deducir que las estrategias de la CIM hoy en día, están impulsadas principalmente por la tecnología (Vollero et al., 2019). Debido a la digitalización y la tecnología, hoy en día, la comunicación bidireccional es fundamental (Vollero et al., 2019). Por lo general, las empresas realizan una comunicación $\mathrm{y}$, por consiguiente, comienzan el contacto con la audiencia transmitiendo un mensaje (Bacile et al., 2014). Sin embargo, debido a los medios digitales, los consumidores interactúan con las organizaciones cada vez más. Por ello, actualmente las organizaciones deben realizar un seguimiento de las comunicaciones emitidas por la audiencia, tales como, mensajes de opinión, 
valoraciones, contenidos creados por los usuarios, el boca a boca electrónico (eWOM) (Allsop et al., 2007) y cualquier información que los grupos de interés pueden generar en los diferentes entornos digitales.

Las organizaciones deberían interiorizar dos cuestiones importantes en este sentido. Por un lado, es importante que las organizaciones consideren que los grupos de interés, principalmente los consumidores, son participantes activos en las representaciones de la marca (Aspara et al., 2014). Al mismo tiempo, las organizaciones deben responder a la información que la audiencia genera, es decir, interactuar.

A la hora de interactuar con la audiencia, se recomienda crear las comunicaciones desde la organización, y que estas aporten valor. Estas tienen que ser conscientes de que desde la década de los noventa, después del lanzamiento de la World Wide Web, la audiencia tiene acceso a un sinfín de información sobre la organización. Puede realizar comparaciones de precios de los productos o servicios con los proporcionados por diferentes organizaciones, explorar las características del producto, reseñas, etc. Por ello, al generar comunicaciones para los consumidores en sus redes, es importante aportar un contenido atractivo que no aporte la información a la que la audiencia pueda acceder por sí sola (Ahmad et al., 2016). Es decir, no es recomendable aportar únicamente información acerca de precios o características 'objetivas' (Cao et al., 2020). Sin embargo, puede ser interesante publicar contenidos en relación a experiencias de la audiencia que ha consumido el producto o servicio de la organización en cuestión.

En la década del año 2000, cuando surgieron las redes sociales, estas permitieron a la audiencia crear sus propios contenidos relacionados con las marcas (es decir, documentos audiovisuales 'utilizando' o 'consumiendo' un producto o servicio ya adquirido). Estas personas que realizaban este tipo de publicaciones son conocidas como 'creadoras de contenido', o influencers, en caso de que tengan una gran cantidad de seguidores en sus redes sociales. Lo mencionado anteriormente también generó un cambio en las estrategias de la CIM y el marketing de contenidos cobró cada vez más relevancia. El marketing de contenidos se basa en proporcionar información o contenido de valor acerca de la oferta de la organización que la audiencia no sabe. El contenido es considerado el 'rey' del marketing en estos momentos dado que posibilita atraer a la audiencia y fomenta la interacción por medio de diferentes estrategias concretas como preguntas retóricas y contenido innovador (Müller y Christandl, 2019).

\section{EL SECTOR DE LA EDUCACIÓN SUPERIOR (ES) Y EL MARKETING DE LA EDUCACIÓN SUPERIOR (MES)}

La educación superior (ES) es la formación educativa posterior a la graduación o la educación secundaria, y es proporcionada por instituciones educativas (Saunders, 2015). Hay muchas características que hacen que los temas relacionados con la gestión de la educación superior (ES) sea un desafío, como la variedad y naturaleza de sus grupos de interés, los debates sobre quién es el consumidor (estudiantes, familias o empresas). Asimismo, también existen discrepancias respecto a si es apropiada la aplicación de prácticas orientadas a los negocios o no. Es un sector en auge, en el que la comercialización, la ubicación de las instituciones, las instalaciones de cada campus o las titulaciones ofertadas (Rutter et al., 2017) también son atributos retadores de este tipo de instituciones. Además, se han producido algunos cambios en el ámbito de la ES (Molesworth et al., 2009; Todd et al., 2017), como el constante crecimiento de oferta y la competencia global (Maringe, 2010), la importancia en la empleabilidad de las personas graduadas (McGregor et al., 2002) o la variación en los regímenes de financiación y becas (Foskett, 2011). 
Según Christensen, 'el 50 por cien de las 4.000 facultades y universidades de Estados Unidos estarán en quiebra en 10-15 años’ (2017). Sin embargo, desde la Comisión Europea (https://ec.europa.eu) ha aumentado la inversión pública general y las becas proporcionadas al alumnado para la educación en Europa. No obstante, según el informe 'La Universidad española en cifras', los rectores de las universidades españolas plantean que las plazas ofertadas en las universidades no se llenan y el 27 por cien de las titulaciones tienen menos de 40 alumnos (Hernández y Pérez, 2017). En efecto, el auge de la educación en línea y la oferta diversa de titulaciones entre las distintas universidades e instituciones han creado también una situación competitiva que genera dificultades para cubrir las plazas y en la que las instituciones de educación superior (IES) necesitan diferenciarse para poder atraer estudiantes potenciales a su universidad.

La competencia entre universidades crece. Debido a lo anterior, es muy importante diferenciarse y una de las acciones llevadas a cabo por las universidades está relacionada con realizar acciones de marketing y comunicación interactivas y con mayor impacto en las que se considera el amplio abanico disponible de medios de comunicación. Uno de los enfoques y beneficios de la CIM es que tiene un enfoque interactivo, teniendo en cuenta dicho enfoque y el resto de beneficios que proporciona en relación a un mayor rendimiento en cuanto a ventas, imagen y satisfacción por ejemplo (Porcu et al., 2017), se considera un concepto válido para ser estudiado en el marketing de la educación superior (MES). Y es que los diversos medios de comunicación están a su vez en constante evolución, y por ello, las investigaciones continúan con el objetivo de proponer las estrategias de marketing más adecuadas para la educación superior (MES) (Elken, 2019; Hemsley-Brown, 2016). A principios de la década de los ochenta, el MES surgió en el contexto académico de Estados Unidos (Litten, 1980), sin embargo, no fue hasta finales de la década de los noventa cuando el MES se aplicó especialmente en el contexto europeo.

Hoy en día, las instituciones de educación superior (IES) aplican prácticas de marketing empresarial con diferentes objetivos. Sin embargo, según la literatura, en materia del MES todavía no se ha avanzado lo suficiente debido a varias razones; las instituciones tienen una resistencia interna a la marketización, dificultades para definir su negocio principal y también para decidir si el alumnado debe ser visto como el consumidor en todo momento (Binsardi y Ekwulugo, 2003). De hecho, existen discrepancias sobre si el comportamiento del sector de la educación superior (ES) es similar al del sector de negocios o comercial habitual o no (Hemsley-Brown y Oplatka, 2006; Nicolescu, 2009; Parahoo et al., 2016; Rutter et al., 2017; Temple y Shattock, 2007). En este contexto, y considerando la ES como un servicio, el debate persiste y aún se cuestiona si las teorías aplicables a la comercialización de servicios en el sector de negocios o comercial habitual son adecuadas o no para aplicar en las IES (Hemsley-Brown y Goonawardana, 2007; Ramachandran, 2010).

Sin embargo, para ser más competitivos en el sector de la ES, una de las soluciones podría ser aplicar plenamente las prácticas orientadas al negocio, y así, dar un paso hacia la marketización de la ES, la cual se está produciendo actualmente. En este sentido, una de las estrategias de marketing más exitosas es la formalización de las alianzas entre IES o entre IES y socios comerciales (Hemsley-Brown, 2016). Estas están aumentando debido a los beneficios que aportan en este entorno en constante cambio. Y es que hasta hace muy poco, las IES privadas se preocupaban más por las inscripciones y las actividades de marketing que las públicas. Sin embargo, hoy en día ambos tipos de instituciones realizan acciones de captación y de marketing. Además, dado que la competencia aumenta entre las IES, están adoptando un enfoque más orientado a los negocios y utilizan prácticas más orientadas al sector comercial (Hemsley-Brown, 2016; Veloutsou et al., 2005). Teniendo en cuenta la evolución antes mencionada, deberían tenerse en cuenta los diferentes medios y canales existentes relacionados 
con el marketing empresarial. Debido a que brindan infinidad de beneficios y, por otro lado, son coherentes con la marketización que la educación superior (ES) está viviendo.

Cada vez es más relevante adaptar las estrategias de MES a la complejidad y naturaleza del sector de la ES. Al mismo tiempo, el objetivo final de las estrategias MES es atraer nuevos estudiantes. Sin embargo, las IES, tienen varios grupos de interés y todos tienen una gran influencia en la decisión de matriculación. Es por ello que las personas responsables de llevar a cabo las acciones de captación y marketing en la ES trabajan con un reto importante: interactuar y dirigir sus comunicaciones a varios grupos de interés, como familias, antiguos alumnos o empresas.

En definitiva, en estas organizaciones que brindan educación a su estudiantado y además interactúan con otros grupos de interés, es necesario llevar a cabo la marketización de manera cuidadosa (Gibbs, 2011).

\section{CIM APLICADA A LA ES DURANTE LA PANDEMIA DEBIDO A LA COVID-} 19

Teniendo en cuenta la naturaleza del sector de la ES, las IES deben dar una respuesta rápida (e interactuar) utilizando todas las vías y medios de comunicación a su disposición (a veces de forma remota), y en ocasiones, bajo el efecto de cuestiones globales (como la pandemia mundial debido a la COVID-19 en 2020). Además, las IES deben dar respuesta a todos los grupos de interés y principalmente, al alumnado.

Hayes (2007), sugirió que la CIM podría ser adecuada en el MES, ya que es un concepto bien aceptado en el contexto académico y profesional, y utiliza todos los medios de comunicación y marketing (online y offline) accesibles para mantener la relación con la audiencia fomentando la interacción. Dado que las IES reciben varias demandas de información a través de diferentes canales (Alcaide-Pulido et al., 2017), Clark et al. (2017) sugieren que las redes sociales (medio digital) son clave para las instituciones, ya que promueven la interacción y, por consiguiente, las relaciones a largo plazo con los grupos de interés. Sin embargo, se deben considerar las acciones e información recibida por todos los medios, como jornadas de puertas abiertas, información impresa, etc. Y promover también la interacción en estos, por ejemplo, haciendo un posterior contacto o seguimiento con la audiencia. En consecuencia, es recomendable una estrategia de comunicación y marketing estable y, según varios académicos y profesionales, la CIM es el mejor enfoque para gestionar con éxito diversos medios (Ismail et al., 2017).

Sin lugar a dudas, todo proceso que pueda fortalecer el MES es beneficioso para el sector de la ES (Gordon-Isasi et al., 2020). Fomentar el diálogo continuo (comunicación bidireccional) y las relaciones a largo plazo (Porcu et al., 2017) es crucial para la audiencia del contexto de la ES y estas son algunas de las bases de las estrategias de la CIM. No obstante, son escasas las propuestas que analizan la implementación de la CIM (Manoli y Hodgkinson, 2019) y evalúan los beneficios que brinda el constructo (Porcu et al., 2019).

Además, la literatura sobre CIM en el contexto de la ES también es escasa. Ha habido algunas propuestas que exploran las maneras en las que se ha tomado conciencia sobre la CIM (Estaswara, 2016) y han analizado el proceso de aplicación de la misma en la ES. Otra propuesta sugirió algunos indicadores de mejora en la enseñanza en relación a la CIM teniendo en cuenta la percepción del profesorado (Kerr y Kelly, 2017), también se publicaron una decena de artículos sobre cómo introducir la CIM en el currículum docente (Šerić, 2016). Se han presentado ponencias en conferencias (Porcu et al., 2012). También se ha publicado alguna 
propuesta sobre la implementación de la CIM en algunas IES de Malasia (Chu Abdullah y Bin Mohamad, 2015).

La publicación de Gordon-Isasi et al. (2020), expone la CIM como estrategia para el sector en cuestión y también una escala de medición para poder medir el constructo y auditar los esfuerzos en materia de marketing y comunicación. Los autores concluyen que es recomendable seguir investigando la temática y medir si la CIM y los rendimientos asociados a las instituciones están positivamente relacionados (ya que así lo concluyen diversas propuestas académicas). Otra de las publicaciones que mide el efecto beneficioso de la CIM en el sector de la educación superior es la investigación llevada a cabo por Edmiston (2009). En la anterior investigación se midió si las instituciones de educación superior habían avanzado hacía la integración efectiva de los procesos CIM y también analizó cómo se estructuraban los procesos de marketing y comunicación en las universidades. Los resultados indicaron que una estrategia CIM fortalecía la marca de las universidades. En definitiva, las escasas publicaciones concluyen que la CIM tiene un efecto positivo en las estrategias relacionadas con el MES, particularmente en la interacción y cultivo de relaciones con sus estudiantes y grupos de interés (Porcu et al, 2019).

Cuando se desarrollan estrategias de marketing en contextos de ES, los temas globales también son un desafío a considerar. Ejemplo de ello es el brote de enfermedad por coronavirus (un brote de neumonía viral), COVID-19, que desde diciembre de 2019 se ha extendido por todo el mundo y se ha declarado pandemia en marzo de 2020. Este brote ha supuesto que se cerraran IES en 185 países, afectando a 1.542.412.000 estudiantes, que constituyen el 89,4 por cien del total de estudiantado matriculado (Marinoni et al., 2020). Tras el brote de coronavirus, han surgido diferentes desafíos. En primer lugar, en relación a los flujos de comunicación claros y efectivos con el personal y los estudiantes. Estos han cobrado una gran relevancia. La comunicación en relación a las medidas y el modus operandi de las IES con todos los grupos de interés también ha sido muy relevante durante la pandemia. A su vez, si esta transmisión de la información se ha hecho de manera adecuada y se ha interactuado adecuadamente, habrá ayudado a mantener las relaciones a largo plazo que se han establecido a lo largo de los años.

En segundo lugar, en relación a la velocidad de actuación de las IES, estas tenían que actuar con rapidez. En cuanto a la modalidad en la que se iba a realizar la enseñanza, por ejemplo, las sesiones en el aula debían ser reemplazadas por el aprendizaje a distancia. Se puede deducir, por tanto, que la rápida adaptación de las IES a los cambios es un aspecto muy relevante. En el caso de la pandemia antes mencionada, la Universidad de Cambridge fue, hasta donde sabemos, la primera universidad que comunicó que todas las sesiones del próximo curso 2020-21 iban a ser impartidas on-line (BBC News, 2020, 19 de mayo). Cuando el alumnado potencial toma una decisión sobre su carrera universitaria, estas respuestas rápidas, la adaptación y las comunicaciones interactivas (el diálogo) son clave. Comunicar de manera ágil y clara ayuda crear una oportunidad a partir de una dificultad o reto.

Es destacable que, en estas situaciones de incertidumbre, la decisión rápida y su comunicación por parte de las organizaciones, se considera una buena práctica. Para conseguirlo, podría considerarse y ser estudiado un enfoque como la CIM, donde se fomenta la interacción por medio de diferentes medios de comunicación. Gracias a estas estrategias podrían reducirse las situaciones de incertidumbre (una situación generalmente incómoda) del futuro y actual estudiantado. Las estrategias basadas en CIM permiten también llevar a cabo acciones de manera rápida y ágil, y esto permitiría a la audiencia tomar una decisión lo antes posible acerca de su aprendizaje universitario. En este caso concreto, puede afectar la toma de decisiones del futuro alumnado acerca de su carrera universitaria, lo que a su vez genera un impacto negativo en las cifras de matrículas nacionales, y también en las internacionales. Algunas IES, especialmente las privadas, podrían tener consecuencias financieras negativas, 
Gordon-Isasi, J., Narvaiza Cantin, L., Gibaja Martíns, J. J.

La comunicación integrada de marketing (CIM) en la educacion superior (ES)

que podrían reducirse con una estrategia basada en el enfoque de la CIM en la que se fomenten las interacciones, rápida toma de decisiones y comunicación a todas sus partes interesadas.

\section{DISCUSIÓN}

Las definiciones propuestas sobre la CIM concluyen que desde su primera definición pretende abarcar, armonizar y alinear todos los medios y actividades de comunicación de una organización. No obstante, la CIM se ha ido adaptando durante estos años a las diferentes y cambiantes realidades. Así, se han propuesto varias definiciones a lo largo de los años (entre 1989 y 2017), también dimensiones y, además, se han introducido diferentes elementos en sus propuestas. Es relevante destacar que hasta 2017 la interacción en las estrategias CIM no tenía tanto protagonismo y eso puede ser debido a que los medios digitales actuales (redes sociales) permiten la comunicación bidireccional de manera más ágil en comparación con los medios más tradicionales (prensa impresa, radio, televisión, etc.). Actualmente, la CIM se considera una ventaja competitiva para las organizaciones (Butkouskaya et al., 2019) pero en el sector de la educación superior (ES) en particular, está poco estudiada. Sin embargo, en el escenario de comunicación y marketing actual, volátil y cambiante, un concepto como la CIM que gestiona cuidadosamente las comunicaciones y las interacciones con la audiencia es muy interesante. En concreto en el sector de la ES, los especialistas en marketing deben estar al día respecto a los nuevos medios que utiliza la audiencia y aprender constantemente a desarrollar mensajes efectivos que se transmitirán en ellos.

Por otro lado, muchas publicaciones en materia de la CIM continúan con un enfoque más tradicional sobre el concepto, sin considerar plenamente el enfoque digital, lo que supone un riesgo de quedarse atrás. Alternativamente, como acaba de suceder en varias organizaciones proactivas, se podría adoptar una gestión gerencial (Vollero et al., 2019). Es decir, dado que la gestión del marketing y comunicación es una labor primordial para una organización, se podría plantear a niveles directivos de la misma, la gestión clave de acciones a llevar a cabo centradas en fomentar la interacción con la audiencia.

Además, considerando los efectos generados debido a pandemias mundiales, estas actividades enmarcadas en la estrategia de la CIM cobran mayor importancia. La audiencia tiene que estar mejor informada que nunca en materia del modus operandi de la organización. ¿Cómo se van a realizar las comunicaciones y/o ventas del producto/servicio? ¿De manera remota? ¿de manera presencial? ¿se cesarán las actividades de cierta oferta? Cada IES es diferente, debido a su tamaño, ubicación u oferta, pero es importante que se trabaje en un plan de marketing y comunicación en el que la interacción sea primordial. La interacción permitirá informar a los grupos de interés de todas y cada una de las decisiones tomadas en tiempos de incertidumbre. Por otro lado, como acción para fomentar la interacción y como ejemplo de estrategia de marketing de contenidos, en el caso de la educación superior (ES), una estrategia de marketing de contenidos para comunicar un máster de marketing digital, podría ser una comunicación en los medios en la que se explican las diferentes estrategias que se pueden llevar a cabo como ejemplo (Google Ads, Display, SEO, SEM, Email Marketing, etc.). De esta manera se aporta un conocimiento a la audiencia que genera una interacción (puede responder a esta publicación mostrando intéres) y se explica detalladamente en qué consiste dicho programa que se está intentando comunicar.

La principal limitación de este trabajo se refiere a la generalizabilidad de los resultados, debido al contexto específico del estudio, el de la educación superior (ES), las estrategias llevadas a cabo en materia de la CIM son difícilmente extrapolables a las diferentes instituciones de educación superior (IES). Es difícil generalizar y proponer una estrategia de 
CIM efectiva para fomentar la interacción en tiempos de pandemia con los diferentes grupos de interés y en las diferentes instituciones existentes. Por lo tanto, se necesita investigación futura para realizar estudios en el marco de la CIM en diversas áreas geográficas e IES. Esto permitirá corroborar la validez del enfoque y contribuir a construir un marketing en la educación superior (MES) basado en la CIM el cual fomente el diálogo con la audiencia.

Además, como futura investigación, cabe destacar que la CIM es importante y útil en el mercado actual (Kitchen, 2017), pero su implementación y contribución organizacional requieren atención (Patti et al., 2017). Por ello, se recomienda seguir trabajando en propuestas empíricas acerca del constructo CIM (Manoli y Hodgkinson, 2019) aplicado a diferentes contextos. Estas propuestas empíricas permitirán recopilar datos y diferentes interacciones realizadas en organizaciones que apliquen la CIM y ayudarán a conocer las estrategias más efectivas para a posteriori poder aplicarlas en diferentes espacios. Al mismo tiempo, se requiere profundizar en la investigación con el objetivo de proponer orientación sobre la CIM a las personas responsables de marketing. El objetivo primordial sería sugerir una serie de directrices con el propósito de gestionar de manera eficiente el entorno omnicanal en el que navegan, un entorno digital e interactivo y también el no digital.

También se recomiendan futuras investigaciones en materia de la CIM bajo los impactos de diferentes sucesos globales, como la pandemia mundial generada debido al COVID-19. Además, se propone también seguir trabajando en el concepto de la CIM desde un punto de vista digital, considerando los medios digitales en las estrategias e introduciendo la interacción por medio de la digitalidad. Finalmente, las investigaciones con guías, pautas y recursos para poder realizar las estrategias e interacciones en los diferentes medios en los que está presente la organización, podrían ser de mucha ayuda para los profesionales.

\section{CONCLUSIONES}

Este trabajo comienza exponiendo la evolución del concepto de la CIM desde que se inició a finales de los ochenta. Además, también responde a las llamadas acerca de profundizar en la investigación en el campo de la CIM aplicada a diferentes contextos (Šerić, 2017), en este caso, al contexto de la educación superior (ES), en el cual las propuestas son escasas. Finalmente, también brinda una breve exposición acerca del estado actual del marketing en la educación superior (MES) bajo los efectos de la pandemia debido al COVID-19 desde un enfoque de la CIM.

Teniendo en cuenta el abanico de medios disponibles, digitales y no digitales, el presente artículo propone estudiar las estrategias más adecuadas para fomentar la interacción con la audiencia. Considerando los tiempos de pandemia actuales, se sugiere hacer hincapié en medios digitales dado que la prespecialidad (tanto para las sesiones lectivas como para las acciones de marketing como eventos, puertas abiertas, etc.) es cada vez más retadora. Para poder llevar a cabo una estrategia CIM digital lo más beneficiosa posible, se recomienda planificar y medir cada esfuerzo de manera efectiva.

En cuanto a la medición de la CIM, sigue siendo un tema de discusión, ya que no existe un sistema de medición definitivo todavía. Sin embargo, existen varias propuestas en la literatura, y en el caso de la educación superior, para poder medir los esfuerzos y resultados de las estrategias CIM, se recomienda utilizar herramientas o escalas específicas, como la propuesta por Gordon-Isasi et al., (2020) que mide y alcanza como resultado de medición el nivel de CIM obtenido en una institución de educación superior (IES). 


\section{BIBLIOGRAFÍA}

Ahmad, N. S., Musa, R., y Harun, M. H. M. (2016): The impact of social media content marketing (SMCM) towards brand health. Procedia Economics and Finance, $\mathrm{n}^{\mathrm{0}}$ 37, pp. 331-336. doi:10.1016/s2212-5671(16)30133-2

Alcaide-Pulido, P., Alves, H., y Gutiérrez-Villar, B. (2017): Development of a model to analyze HEI image: A case based on a private and a public university. Journal of Marketing for Higher Education, vol. 27, n 2, pp. 162. doi:10.1080/08841241.2017.1388330

Allsop, D. T., Bassett, B. R., y Hoskins, J. A. (2007): Word-of-mouth research: Principles and applications. Journal of Advertising Research, vol. 47, n 4, pp. 398-411.

Aspara, J., Aula, H., Tienari, J., y Tikkanen, H. (2014): Struggles in organizational attempts to adopt new branding logics: The case of a marketizing university. Consumption Markets y Culture, vol. 17, no 6, pp. 522-552. doi:10.1080/10253866.2013.876347

Bacile, T. J., Ye, C., y Swilley, E. (2014): From firm-controlled to consumer-contributed: Consumer co-production of personal media marketing communication. Journal of Interactive Marketing, vol. 28, $\mathrm{n}^{\circ}$ 2, pp. 117-33.

Binsardi, A., y Ekwulugo, F. (2003): International marketing of british education: Research on the students' perception and the UK market penetration. Marketing Intelligence $y$ Planning, vol. 21, $\mathrm{n}^{\mathrm{o}}$ 5, pp. 318-327. Retrieved from https://www.emerald.com/insight/content/doi/10.1108/02634500310490265/full/html

Butkouskaya, V., Llonch-Andreu, J., y Alarcón-Del-Amo, M. (2019): Strategic antecedents and organisational consequences of IMC in different economy types. Journal of Marketing Communications, 1-22. doi:10.1080/13527266.2019.1633551

Cao, X., Zhang, Y., Feng, X., y Meng, X. (2020): Investor interaction and price efficiency: Evidence from social media. Finance Research Letters, 101747. doi:https://doi.org/10.1016/j.frl.2020.101747

Caywood, C., Schultz, D., y Wang, P. (1991): Integrated marketing communication: A survey of national consumer goods. Northwestern University: Evanston, IL: Department of Integrated Marketing Communication.

Christensen, C.50 por cien of US universities will be bankrupt in 10-15 years. Paper presented at the Innovation + Disruption Symposium in Higher Education,

Chu Abdullah, S. A., y Bin Mohamad, M. Y.Integrated marketing communication (IMC): A study of selected malaysian institutions of higher education (IHEs). Paper presented at the International Conference on Multidisciplinary Researchynbsp;

Clark, M., Fine, M. B., y Scheuer, C. (2017): Relationship quality in higher education marketing: The role of social media engagement. Journal of Marketing for Higher Education, vol. 27, $\mathrm{n}^{\mathrm{o}}$ 1, pp. 40-58. doi:10.1080/08841241.2016.1269036

Daszkiewicz, M., y Pukas, A. (2016): Integrated marketing communication - towards a holistic concept doi:10.15611/noz.2016.3.02

Duncan, T. R., y Moriarty, S. (1998): A communication-based marketing model for managing relationships. Journal of Marketing, vol. 62, n 2, pp. 1-13.

Duncan, T., y Caywood, C. (1996): The concept, process, and evolution of integrated marketing communications. In E. Thorson, $\mathrm{y}$ J. Moore (Eds.), Integrated communication: Synergy of persuasive voices (pp. 13-34). Mahwah, NJ: Lawrence Erlbaum,

Duncan, T. (1994): New sides of IMC. In F. Corrine, y K. Lisa (Eds.), Marketitig communication strategies fodav and tomorrow: Integration, allocation, and interactive technologies (pp. Marketing Science Institute Report $\mathrm{n}^{\circ}$. 94-109). Cambridge, MA: Marketing Science Institute. 
Duncan, T., and S.E. Moriarty. 2006. How integrated marketing communication's 'touchpoints' can operationalize the service-dominant logic. In The service-dominant logic of marketing: Dialog, debate, and directions, ed. R.F. Lusch and S.L. Vargo, pp. 236-249. London: Routledge.

Eagle, L., y Brennan, R. (2007): Are students customers? TQM and marketing perspectives. Quality Assurance in Education, vol. 15, $\mathrm{n}^{\circ}$ 1, pp. 44-60. doi:10.1108/09684880710723025

Edmiston-Strasser, D. M. (2009): An examination of integrated marketing communication in U.S. public institutions of higher education. Journal of Marketing for Higher Education, vol. 19, no 2, pp. 142-165. doi:10.1080/08841240903423166

Elken, M. (2019): Marketing in higher education. Encyclopedia of international higher education systems and institutions (pp. 1-5). Dordrecht: Springer Netherlands. doi:10.1007/978-94-017-9553-1_569-1 Retrieved from https://doi.org/10.1007/978-94017-9553-1 569-1

Estaswara, H. (2016): Integrated marketing communications (imc) in higher education in indonesia. Polish Journal of Management Studies, vol. 14, $\mathrm{n}^{\circ} 1$, pp. 74-83. doi:10.17512/pjms.2016.14.1.07

Foskett, N. H. (2011): Markets, government, funding and the marketisation of UK higher education, In M. Molesworth, R. Scullion y E. Nixon (Eds.), The marketisation of higher education and the student consumer (pp. 25-38). Abingdon: Routledge.

Gibbs, P. (2011): An aristotelian model for ethical higher education marketing. Journal of Marketing for Higher Education, vol. 21, $\mathrm{n}^{\mathrm{o}}$ 2, pp. 203-214. doi:10.1080/08841241.2011.623732

Gordon-Isasi, J., Narvaiza, L., y Gibaja, J. J. (2020): Revisiting integrated marketing communication (IMC): A scale to assess IMC in higher education (HE). Journal of Marketing for Higher Education, 1-33. doi:10.1080/08841241.2020.1758283

Hayes, T. (2007): Delphi study of the future of marketing of higher education. Journal of Business Research, vol. 60, no 9, pp. 927-931.

Hemsley-Brown, J., y Oplatka, I. (2006): Universities in a competitive global marketplace. International Journal of Public Sector Management, vol. 19, $\mathrm{n}^{\circ} 4$, pp. 316-338. doi:10.1108/09513550610669176

Hemsley-Brown, J., y Goonawardana, S. (2007): Brand harmonization in the international higher education market. Journal of Business Research, vol. 60, $\mathrm{n}^{\circ}$ 9, pp. 948. doi:10.1016/j.jbusres.2007.01.019

Hemsley-Brown, J. (2016): Exploring brand identity, meaning, image, and reputation (BIMIR) in higher education. Journal of Business Research, vol. 69, n ${ }^{\circ}$ 8, pp. 3019-3022. Retrieved from http://www.econis.eu/PPNSET?PPN=863737994

Hernández, J. y Pérez, J. A. (2017): A UN1VERSIDAD ESPAÑOLA 3N CIFR4S [Spanish University in figures] Retrieved from CRUE website: http://www.crue.org/SitePages/La-Universidad-Espa por cienC3 por cienB1ola-enCifras.aspx

Ismail, S. Z., Heng, K. T., Mohamed, F. M., y Anwar, H. (2017): Integrated marketing communication in the era of online environment. International Journal of Business and Management Invention, vol. 6, $\mathrm{n}^{0}$ 12, pp. 12-14.

Kerr, G., y Kelly, L. (2017): IMC education and digital disruption. European Journal of Marketing, 51(3), 406-420. Retrieved from Permanent link to this document: http://dx.doi.org/10.1108/EJM-08-2015-0603

Kerr, G., Schultz, D., Patti, C., y Kim, I. (2008): An inside-out approach to integrated marketing communication. International Journal of Advertising, vol. 27, $\mathrm{n}^{\circ} 4$, pp 511. doi:10.2501/S0265048708080098 
Kitchen, P. J. (2017a): Guest editorial. European Journal of Marketing, vol. 51, nº 3, pp. 394405.

Kitchen, P. J. (2017b): Guest editorial. European Journal of Marketing, vol. 51, nº 3, pp. 394405. doi:10.1108/EJM-06-2016-0362

Kliatchko, J. (2005): Towards a new definition of integrated marketing communications. International Journal of Advertising, vol. 24, $\mathrm{n}^{0}$ 1, pp. 7. Retrieved from http://search.proquest.com/docview/231107477

Kliatchko, J. (2008): Revisiting the IMC construct. International Journal of Advertising, vol. 27, $\mathrm{n}^{\mathrm{o}}$ 1, pp. 133. doi:10.1080/02650487.2008.11073043

Kliatchko, J., y Schultz, D. E. (2014): Twenty years of IMC. International Journal of Advertising, vol. 33, $\mathrm{n}^{\circ}$ 2, pp. 373. doi:10.2501/IJA-33-2-373-390

Litten, L. H. (1980): Marketing higher education: Benefits and risks for the american academic system. The Journal of Higher Education, vol. 51, $\mathrm{n}^{\circ}$ 1, pp. 40-59.

Luxton, S., Reid, M., y Mavondo, F. (2017): IMC capability: Antecedents and implications for brand performance. European Journal of Marketing, vol. 51, no 3, pp. 421-444. doi:10.1108/EJM-08-2015-0583

Manoli, A. E., y Hodgkinson, I. R. (2019): The implementation of integrated marketing communication (IMC): Evidence from professional football clubs in england. Journal of Strategic Marketing, vol. 28, n ${ }^{\circ}$ 6, pp. 1-22. doi:10.1080/0965254x.2019.1593225

Maringe, F. (2010): The meanings of globalization and internationalization in HE: Findings from a world survey., pp. 17-34.

Marinoni, G., van't Land, H. y Jensen, T. (2020): The impact of covid-19 on higher education around the world. International Association of Universities (IAU). Global Survey Report ISBN: 978-92-9002-212-1

Marketing Science Institute (2016): Marketing Institute of Science. 2016-2018 Research Priorities. RP7: Developing and Delivering Fully Integrated Marketing Programs. Retrieved from: https://www.msi.org/research/2014-2016-research-priorities/

Marketing Science Institute (2018): Research Priorities 2018-2020. Cambridge, Mass.: Marketing Science Institute.

Marketing Science Institute (2020): Marketing Institute of Science. Marketing Topics. Priority Topics. The Rise of Omnichannel Promotion and Distribution. Retrieved from: https://www.msi.org/topics/the-rise-of-omnichannel-promotion-and-distribution/

McGregor, P., Thanki, R., y McKee, P. (2002): Home and away: Graduate experience from a regional perspective. Applied Economics, vol. 34, n 2, pp. 219-230.

Molesworth, M., Nixon, E., y Scullion, R. (2009): Having, being and higher education: The marketisation of the university and the transformation of the student into consumer. Teaching in Higher Education, vol. 14, $\mathrm{n}^{\mathrm{o}}$ 3, pp. 277-287. doi:10.1080/13562510902898841

Moriarty, S. E., y Schultz, D. E. (2012): Four theories of how IMC works. in advertising theory, ed. S. rodgers and E. thorson, 491505. New York: Routledge.

Müller, J., y Christandl, F. (2019): Content is king - but who is the king of kings? the effect of content marketing, sponsored content y user-generated content on brand responses. Computers in Human Behavior, 96, 46-55. doi:https://doi.org/10.1016/j.chb.2019.02.006

Nicolescu, L. (2009): Applying marketing to higher education: Scope and limits. Management y Marketing, vol. 4, $\mathrm{n}^{\mathrm{o}} 2$ Summer, Retrieved from https://www.openaire.eu/search/publication?articleId=od__645::52bc93732a4be 19e8c6455a9e4e96fae 
Nowak, G. J., y Phelps, J. (1994): Conceptualizing the integrated marketing communications' phenomenon: An examination of its impact on advertising practices and its implications for advertising research. Journal of Current Issues y Research in Advertising, vol. 16, $\mathrm{n}^{\circ}$ 1, pp. 49-66. doi:10.1080/10641734.1994.10505012

Parahoo, S. K., Santally, M. I., Rajabalee, Y., y Harvey, H. L. (2016): Designing a predictive model of student satisfaction in online learning. Journal of Marketing for Higher Education, vol. 26, $\mathrm{n}^{\mathrm{o}}$ 1, pp. 1-19. doi:10.1080/08841241.2015.1083511

Patti, C. H., Hartley, S. W., van Dessel, M. M., y Baack, D. W. (2017): Improving integrated marketing communications practices: A comparison of objectives and results. Journal of Marketing Communications, vol. 23, $\mathrm{n}^{\mathrm{o}}$ 4, pp. 351-20. doi:10.1080/13527266.2015.1027251

Percy, L. (2008): Strategic integrated marketing communications: Theory and practice. Burlington: Taylor y Francis Group.

Porcu, L., del Barrio-García, S., y Kitchen, P. (2012): How integrated marketing communications (IMC) works? A theoretical review and an analysis of its main drivers and effects. Communication y Society, vol. 25, $\mathrm{n}^{\mathrm{o}}$ 1, pp. 313-348. doi:10.1080/10496491003595726

Porcu, L., Del Barrio-García, S., Rodríguez-Priego, N., y Crespo-Almendros. (2012): Higher education institutions (HEIs) and integrated marketing communications (IMC): An odd couple. Paper presented at the 6th International Technology. Education and Development Conference. [Google Scholar]

Porcu, L., Del Barrio-García, S., y Kitchen, P. (2017): Measuring integrated marketing communication by taking a broad organisational approach: The firm-wide IMC scale. European Journal of Marketing, doi:10.1108/EJM-08-2015-0587

Porcu, L., del Barrio-García, S., Alcántara-Pilar, J. M., y Crespo-Almendros, E. (2019): Analyzing the influence of firm-wide integrated marketing communication on market performance in the hospitality industry. International Journal of Hospitality Management, ${ }^{\circ}$ 80, pp. 13-24. doi:10.1016/j.ijhm.2019.01.008

Ramachandran, N. T. (2010): Marketing framework in higher education. International Journal of Educational Management, vol. 24, $\mathrm{n}^{\mathrm{0}}$ 6, pp. 544-556. doi:10.1108/09513541011067700

Reid, M. (2005): Performance auditing of integrated marketing communication (IMC) actions and outcomes. Journal of Advertising, vol. 34, $\mathrm{n}^{\circ}$ 4, pp. 41-54. Retrieved from http://www.jstor.org/stable/4189318

Rutter, R., Lettice, F., y Nadeau, J. (2017): Brand personality in higher education: Anthropomorphized university marketing communications. Journal of Marketing for Higher Education, vol. 27, no 1, pp. 19-21. doi:10.1080/08841241.2016.1213346

Saunders, D. B. (2015): They do not buy it: Exploring the extent to which entering first-year students view themselves as customers. Journal of Marketing for Higher Education, vol. 25, n ${ }^{\circ}$ 1, Retrieved from DOI: 10.1080/08841241.2014.969798

Schultz, D. E. (1991): Integrated marketing communications: The status of integrated marketing communications programs in the US. Journal of Promotion Management, vol. 1, n 1 , pp. 99-104.

Schultz, D., y Schultz, H. (1998): Transitioning marketing communication into the twenty-first century. Journal of Marketing Communications, vol. 4, $\mathrm{n}^{\mathrm{o}}$ 1, pp. 9-26. doi:10.1080/135272698345852

Schultz, D., y Schultz, H. (2003): IMC, the next generation: Five steps for delivering value and measuring financial returns 
Schultz, D. E., y Patti, C. H. (2009): The evolution of IMC: IMC in a customer-driven marketplace. Journal of Marketing Communications, vol. 15, $\mathrm{n}^{\circ}$ 2-3, pp. 75-84. doi:10.1080/13527260902757480

Šerić, M. y Gil-Saura, I. (2012): ICT, IMC, and Brand Equity in HighQuality Hotels of Dalmatia: An Analysis From Guest Perceptions, Journal of Hospitality Marketing y Management, 21:8, 821-851, DOI: 10.1080/19368623.2012.633211

Šerić, M. (2016): Content analysis of the empirical research on IMC from 2000 to 2015. Journal of Marketing Communications, pp. 1-39. doi:10.1080/13527266.2016.1184708

Šerić, M. (2017): Relationships between social web, IMC and overall brand equity: An empirical examination from the cross-cultural perspective. European Journal of Marketing, vol. 51, n ${ }^{0}$ 3, Retrieved from DOI: 10.1108/EJM-08-2015-0613

Šeric, M., Gil-Saura, I., y Ozretić-Došen, Đ. (2015): Insights on integrated marketing communications: Implementation and impact in hotel companies. Int J Contemp Hospitality Mngt, vol. 27, no 5, pp. 958-979. doi:10.1108/IJCHM-12-2013-0568

Syahputra. (2012): Integrated marketing communications and marketing performances of small medium-sized enterprises (A study on food and beverages SMEs, northern malaysia). Retrieved from http://etd.uum.edu.my/3216/

Taylor, C. R. (2010): Integrated marketing communications in 2010 and beyond. International Journal of Advertising: The Review of Marketing Communications, vol. 29, $\mathrm{n}^{\mathrm{o}}$ 2, $\mathrm{pp}$. 161-164. Retrieved from http://dx.doi.org/10.2501/S0265048710201105

Temple, P., y Shattock, M. (2007): What does "branding" mean in higher education? In B. Stensaker, y V. D’Andrea (Eds.), Branding in higher education. exploring an emerging phenomenon (pp. 73-82) EAIR Series Research, Policy and Practice in Higher Education.

Todd, S., Barnoff, L., Moffatt, K., Panitch, M., Parada, H., y Strumm, B. (2017): A social work re-reading of students as consumers. Social Work Education, vol. 36, n 5, pp. 542-556. doi:10.1080/02615479.2016.1225712

Veloutsou, C., Paton, A., y Lewis, J. (2005): Consultation and reliability of information sources pertaining to university selection: Some questions answered? International Journal of Educational Management, vol. 19, nº 4, pp. 279-291.

Vollero, A., Schultz, D. E., y Siano, A. (2019): IMC in digitally-empowering contexts: The emerging role of negotiated brands. International Journal of Advertising, vol. 38, $\mathrm{n}^{\circ} 3$, pp. 428-449. doi:10.1080/02650487.2018.1535221

William A. Cook. (2004): Editorial: IMC's fuzzy picture. Journal of Advertising Research, vol. $44, n^{\circ} 1$, pp. 1-2. 